\title{
Improving the Quality of Scientific Publications for Revista Urología Colombiana
}

\section{Mejorando la calidad de la publicación científica en la Revista Urología Colombiana}

\author{
Anthony A. Caldamone, MD ${ }^{1}$ \\ ${ }^{1}$ Editor-in-chief, Journal of Pediatric Urology, Rhode Island, USA \\ Urol Colomb 2018;27:199-200.
}

Address for correspondence Anthony A. Caldamone, MD, Editor of Journal of Pediatric Urology, Rhode Island, USA (e-mail: ACaldamone@Lifespan.org).
One of the first scientific periodicals was the Philosophical Transactions, by the Royal Society of London. Its inaugural issue was in 1665 . It was quite prestigious to have a publication in this journal, which lead to authors submitting works of fiction. As a result, a peer-review process was launched in 1752 , establishing standards for the works published in this journal. Prior to this, whether or not a manuscript would be published in this journal was up to the editor's discretion. Other leading publications followed suit, with the British Medical Journal requiring submissions to be peer-reviewed in 1893, and the Lancet in 1976. The peer-review process is not without its intrinsic flaws. However, it has proven to be the fairest way to establish and elevate the level of publications regarding their scientific design, interpretation of data, and conclusions. The editor's role is that of the unbiased judge and jury, and to sort out potential reviewer biases. The goals of the peer-review process would be to ensure that high-quality research is published by improving sub-quality manuscripts and to identify fraud, data manipulation, and duplication. Through this process, the journal would be able to disseminate relevant findings to an interested readership. Overall, the system has accomplished these goals.

There are certain expectations that an author should have regarding the peer-review process. Reviewers should define the important strengths, weaknesses and limitations of the particular study. A peer-review should also state whether and how the manuscript adds to the existing literature as well as identify duplication with other works. In addition, for clinical manuscripts, the review should state whether the findings are clinically relevant, and not just scientifically or statistically relevant.

While quality peer-review establishes a standard for scientific publications, other factors have also been shown to increase the quality of submitted manuscripts as well as the citations of published manuscripts. One of these is the templated or structured review. This is a guide for reviewers to provide more consistent constructive feedback to the authors in an organized format. In a study by Sosa et al $^{1}$ of manuscripts randomized to a structured review, there was a higher quality review and an increased mean number of citations compared with manuscripts that underwent traditional non-structured reviews. Sosa also concluded that an additional factor that was found to improve the quality of a manuscript was the selective addition of a statistician as a coauthor.

The impact factor has become a metric of the quality of a journal. The impact factor of a journal in which an author publishes may have an effect on the academic promotion of the author and on the ability of the author to secure grant funding. In addition, a higher impact factor can have a positive effect on advertising revenue for a given journal. While the calculation of the impact factor is quite straightforward, there are some variables that can have a significant effect on the results. Some of the variables have the potential for being manipulated to a journal's favor. In addition, one has to appreciate that the potential pool of authors who would reference an article in a particular journal is limited by the subject matter of the journal. In pediatric urology, for instance, which is a growing specialty on a relative scale of other disciplines, such as adult urology or general medicine, there is only a limited number of authors who would reference articles from the pediatric urology literature. Concern is also being raised that an editor's decision on a manuscript may be influenced by the predicted effect of the manuscript on the impact factor of the journal. In addition, it has been shown that industry-sponsored trials garner more citations
DOI https://doi.org/ $10.1055 / \mathrm{s}-0038-1667355$. ISSN 0120-789X. eISSN 2027-0119.
Copyright ( 2018, Sociedad Colombiana License terms de Urología. Publicado por Thieme Revinter Publicações Ltda., Rio de Janeiro, Brazil. Todos los derechos reservados. 
than trials funded by a non-profit source. ${ }^{2}$ Therefore, while the impact factor needs to be acknowledged, it does not necessarily reflect the quality of the journal.

Finally, the internet has changed our lives in many ways. This is also true for scientific publications, in that it has allowed for the almost immediate dissemination of information. Open access, which is a concept developed over a decade ago to increase the availability of articles to a larger readership, has been shown to increase citations of a particular manuscript. ${ }^{3}$ What this has led to, however, is a new business model of publishing in which the authors pay a fee for peer-review and open access. This business model could encourage publishers to publish more and more articles to increase the profitability of the journal. In some cases, this leads to a substantial reduction in the quality for the sake of quantity. This has been referred to as predatory publishing. ${ }^{4}$ There have been several reports of completely fabricated articles that have been published in this manner, exposing the lack of quality peerreview. This speaks to the exploitation of authors who are pressured by living in a "publish or perish" culture. The solicitation by these journals, of not only authors but also of reviewers and editors, is robust, just like many of us receive email requests on a daily basis. In fact, the attempt by predatory journals to build an editorial board with a legitimate academic appearance has led to the recruitment of fake editors, fictional characters proposed by academic pranksters. ${ }^{5}$ I refer you to a published urology green list (https://urologygreenlist.wordpress.com) as a guide for appropriate and internationally recognized urological journals, such as this one. "Green" journals allow uploading an accepted manuscript to an open access repository. While there are distinct advantages to open access publishing, as previously mentioned, we must be cautious as to the potential downside that erodes at the very foundation and goal of scientific publication, which is to benefit our patients.

\section{References}

1 Sosa JA, Mehta P, Thomas DC, et al. Evaluating the surgical literature: Can standardized peer-review today predict manuscript impact tomorrow? Ann Surg 2009;250(01):152-158

2 Markovich H. Editors, publishers, impact factors, and reprint income. PLoSMed 2010;7(10):e1000355

3 McKiernan EC, Bourne PE, Brown CT, et al. How open science helps researchers succeed. Elife 2016;5:e16800

4 Beall J. Predatory publishers are corrupting open access. Nature 2012;489(7415):179

5 Sorokowski P, Kulczycki E, Sorokowska A, Pisanski K. Predatory journals recruit fake editor. Nature 2017;543(7646):481-483 